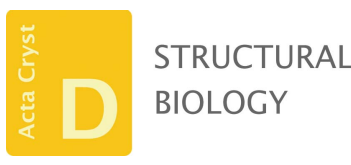

ISSN 2059-7983
Received 14 February 2020

Accepted 5 June 2020

Edited by A. Berghuis, McGill University, Canada

Keywords: FAD-dependent halogenase; crystal soaking; cofactor binding; enzymatic halogenation; bromination; crystal structure.

PDB reference: Xcc4156, 6y1w

Supporting information: this article has supporting information at journals.iucr.org/d
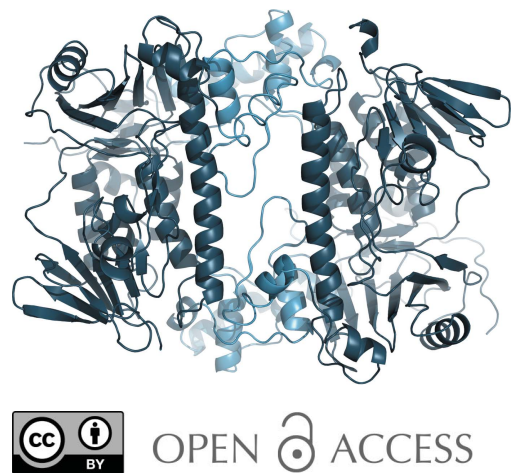

\section{Structure of apo flavin-dependent halogenase Xcc4156 hints at a reason for cofactor-soaking difficulties}

\author{
Christiane Widmann, ${ }^{a}$ Mohamed Ismail, ${ }^{b}$ Norbert Sewald ${ }^{b}$ and Hartmut H. \\ Niemann ${ }^{\mathrm{a} *}$
}

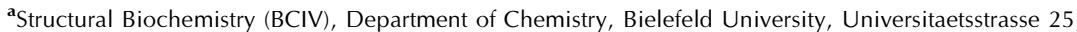
33615 Bielefeld, Germany, and 'brganic and Bioorganic Chemistry (OC III), Department of Chemistry, Bielefeld University, Universitaetsstrasse 25, 33615 Bielefeld, Germany. *Correspondence e-mail: hartmut.niemann@uni-bielefeld.de

Flavin-dependent halogenases regioselectively introduce halide substituents into electron-rich substrates under mild reaction conditions. For the enzyme Xcc4156 from Xanthomonas campestris, the structure of a complex with the cofactor flavin adenine dinucleotide (FAD) and a bromide ion would be of particular interest as this enzyme exclusively brominates model substrates in vitro. Apo Xcc4156 crystals diffracted to $1.6 \AA$ resolution. The structure revealed an open substrate-binding site lacking the loop regions that close off the active site and contribute to substrate binding in tryptophan halogenases. Therefore, Xcc4156 might accept larger substrates, possibly even peptides. Soaking of apo Xcc4156 crystals with FAD led to crumbling of the intergrown crystals. Around half of the crystals soaked with FAD did not diffract, while in the others there was no electron density for FAD. The FAD-binding loop, which changes its conformation between the apo and the FAD-bound form in related enzymes, is involved in a crystal contact in the apo Xcc4156 crystals. The conformational change that is predicted to occur upon FAD binding would disrupt this crystal contact, providing a likely explanation for the destruction of the apo crystals in the presence of FAD. Soaking with only bromide did not result in bromide bound to the catalytic halide-binding site. Simultaneous soaking with FAD and bromide damaged the crystals more severely than soaking with only FAD. Together, these latter two observations suggest that FAD and bromide bind to Xcc4156 with positive cooperativity. Thus, apo Xcc4156 crystals provide functional insight into FAD and bromide binding, even though neither the cofactor nor the halide is visible in the structure.

\section{Introduction}

The activation of $\mathrm{C}-\mathrm{H}$ bonds in electron-rich organic compounds is an important step that enables their use in a range of downstream applications such as metal-catalyzed cross-coupling reactions or modifications via nucleophilic substitution (Runguphan \& O'Connor, 2013; Frese et al., 2016; Durak et al., 2016). Chemical halogenation requires harsh conditions, often employing elemental halogens in combination with Lewis acids. In addition, the process may lead to the formation of byproducts owing to a lack of regioselectivity. Flavin-dependent halogenases (FDHs) have come into focus as a clean alternative because they halogenate their substrates under much milder conditions using halide salts and molecular oxygen at $25^{\circ} \mathrm{C}$ and $\mathrm{pH} 7$ (Keller et al., 2000; Yeh et al., 2005). Their reaction mechanism has been investigated both in vitro (Dong et al., 2005; Yeh et al., 2006, 2007; Flecks et al., 2008; Fraley et al., 2017) and in silico (Fraley et al., 2017; Ainsley 
et al., 2018). FDHs depend on reduced flavin adenine dinucleotide $\left(\mathrm{FADH}_{2}\right)$ as a cofactor, which is bound by a dedicated subdomain. A halide ion $\left(\mathrm{X}^{-}\right)$is coordinated close to the isoalloxazine ring of FAD (Dong et al., 2005). As a first reaction step, FAD-C4a-OOH is formed (Yeh et al., 2006), which subsequently oxidizes the halide ion, resulting in the formation of hypohalous acid (HOX). The HOX then travels around $10 \AA$ to the substrate-binding site of the enzyme, where halogenation takes place (Dong et al., 2005). A conserved active-site lysine plays a crucial role in the halogenation, either via hydrogen bonding to HOX (Flecks et al., 2008) or by forming a lysine haloamine (Yeh et al., 2007). The exact mechanism is still under debate, but the electrophilic aromatic substitution takes place at the substrate $\mathrm{C}$ atom that is closest to the catalytic lysine and, owing to substrate positioning by the enzyme, the reaction is regioselective.

The first FDHs to be characterized were tryptophan halogenases (Trp halogenases; Keller et al., 2000; Yeh et al., 2005). In the following years, a variety of different halogenases were studied that can be grouped into indole halogenases (which include the Trp halogenases; Seibold et al., 2006; Heemstra \& Walsh, 2008; Zeng \& Zhan, 2011; Menon et al., 2016; Ma et al., 2017; Lingkon \& Bellizzi, 2020; Domergue et al., 2019; Luhavaya et al., 2019), phenol halogenases (Buedenbender et al., 2009; Neumann et al., 2010; Zeng et al., 2013; Agarwal et al., 2014; Menon et al., 2017; Mori et al., 2019) and pyrrole halogenases (Hammer et al., 1997; Dorrestein et al., 2005; Yamanaka et al., 2012; Agarwal et al., 2014), depending on the classes of compounds that they halogenate.

Most described indole halogenases belong to the subgroup of Trp halogenases. Using genomic data, we recently identified four halogenases that act on indole derivatives but not on free tryptophan. The native substrates of $\mathrm{BrvH}$, a halogenase identified from a marine metagenome (Neubauer et al., 2018), as well as of three halogenases from Xanthomonas campestris pv. campestris B100 (Ismail et al., 2019), remain unknown. While BrvH preferentially brominated screening substrates, the $X$. campestris (Xcc) halogenases exclusively brominated them. Previously, only Bmp2 and Bmp5, two marine halogenases from Pseudoalteromonas spp., had been shown to selectively incorporate bromide into their substrates (Agarwal et al., 2014). Bmp2 is responsible for the tribromination or tetrabromination of a carrier-tethered pyrrole (El Gamal et al., 2016), whereas Bmp5 catalyzes the bromination and decarboxylation of 4-hydroxybenzoic acid (Agarwal et al., 2014). Both enzymes do not catalyze chlorination, but for Bmp5 in vivo iodination was additionally observed. The exclusive bromination by $\mathrm{Bmp} 2$ was attributed to halide specificity (Thapa et al., 2018). A crystal structure of Bmp2 lacked bound halide and did not reveal the molecular basis of the specificity for bromide (El Gamal et al., 2016). In contrast to brominases that do not chlorinate, several FDHs that natively chlorinate their substrates can also catalyze bromination. Thal and Mpy16, for example, which chlorinate tryptophan and pyrrole, respectively, form brominated products if an excess of bromide is present (Seibold et al., 2006; Thapa et al., 2018). $\mathrm{RebH}$, a Trp halogenase that mediates the chlorination step during rebeccamycin biosynthesis, has been shown to brominate a larger number of non-natural probe substrates than it can chlorinate, leading Lewis and coworkers to suggest that owing to the higher electrophilicity of bromine relative to chlorine in hypohalous acid, a preference for bromination is common in FDHs (Fisher et al., 2019).

We set out to investigate whether the halide specificity of brominases can be attributed to the structural determinants of bromide binding. To this end, we aimed at elucidating the structure of Xcc4156, one of the three brominating Xcc halogenases, in complex with its cofactor and bromide.

\section{Methods}

\subsection{Protein expression}

The $x c c 4156$ gene was amplified from a pET-28a plasmid (Ismail et al., 2019) and cloned into a pETM-11 expression vector which contains a hexahistidine tag followed by a tobacco etch virus (TEV) protease cleavage site. This plasmid was transformed into Escherichia coli BL21 (DE3) pGro7 cells. The pGro7 plasmid (Takara) codes for the expression of the GroEL-GroES chaperone system.

An overnight preculture $\left(30^{\circ} \mathrm{C}, 110 \mathrm{rev} \mathrm{min}^{-1}\right)$ was used to inoculate a 21 culture in LB medium containing $30 \mu \mathrm{g} \mathrm{ml}^{-1}$ kanamycin and $34 \mu \mathrm{g} \mathrm{ml}^{-1}$ chloramphenicol to an optical density at $600 \mathrm{~nm}\left(\mathrm{OD}_{600}\right)$ of 0.1 . The culture was incubated at $37^{\circ} \mathrm{C}\left(90 \mathrm{rev} \mathrm{min}^{-1}\right)$ until an $\mathrm{OD}_{600}$ of 0.4 was reached. $2 \mathrm{~g} \mathrm{l}^{-1}$ L-arabinose and $0.1 \mathrm{~m} M$ isopropyl $\beta$-D-1-thiogalactopyranoside were added for the induction of chaperone and target protein expression, and the cultivation temperature was decreased to $25^{\circ} \mathrm{C}$. The cells were harvested by centrifugation after $20 \mathrm{~h}$ and the resulting cell pellets were washed with phosphate-buffered saline (PBS) and frozen at $-20^{\circ} \mathrm{C}$.

\subsection{Protein purification}

The purification protocol included an immobilized metalaffinity chromatography (IMAC) step followed by ionexchange (IEX) chromatography, TEV digestion and another IMAC step. The exact protocol has been described in Neubauer et al. (2018) and was followed except for the sizeexclusion chromatography step. Protein obtained from the flowthrough and washing steps of the second IMAC step was concentrated using a Vivaspin column (10 000 Da molecularweight cutoff; Sartorius, Germany) to $29.19 \mathrm{mg} \mathrm{ml}^{-1}$ and was stored at $-80^{\circ} \mathrm{C}$ prior to protein characterization and crystallization.

\subsection{Protein characterization}

To analyze whether the protein forms a monomer or a dimer in solution, dynamic light scattering (DLS) as well as size-exclusion chromatography (SEC) were performed.

For DLS, Xcc4156 was diluted to $5 \mathrm{mg} \mathrm{ml}^{-1}$ with $10 \mathrm{mM}$ Tris, $150 \mathrm{~m} M \mathrm{NaCl} \mathrm{pH}$ 8. Samples were centrifuged at $30000 \mathrm{~g}$ for $30 \mathrm{~min}$ prior to analysis and were transferred to a quartz glass cuvette with a path length of $1.5 \mathrm{~mm}$. Measurements were performed using a DynaPro 801 and the molecular 
Table 1

X-ray data-collection and refinement statistics.

The data-collection statistics were taken from the AIMLESS output; the refinement and model statistics were taken from phenix.table_one. For the data sets containing $\mathrm{Br}$, the statistics were calculated treating Friedel mates as different reflections, except for the values marked with an asterisk.

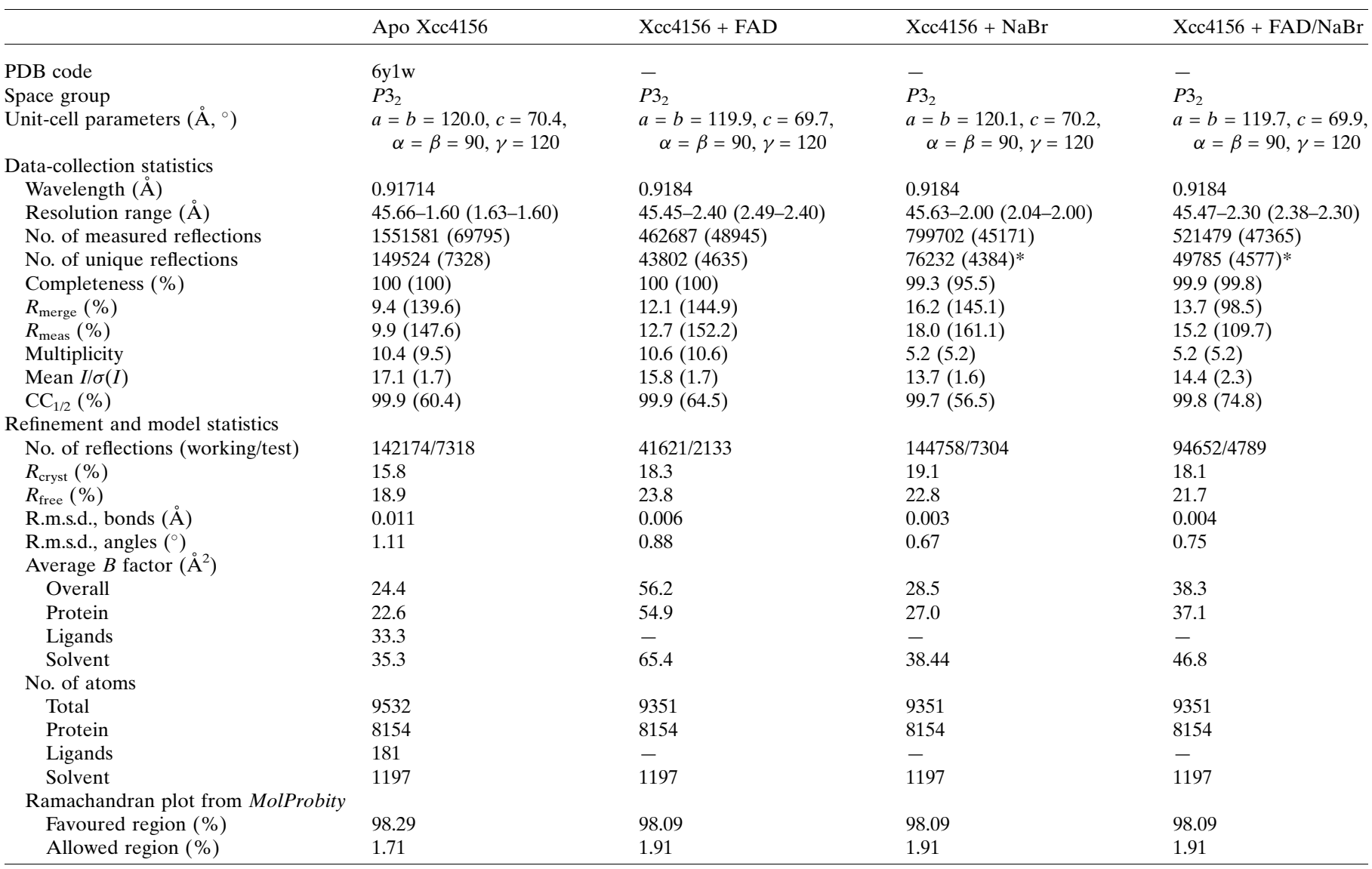

weight was calculated from the hydrodynamic radius using the DYNAMICS software (version 5.25.44). The DLS experiment was performed in triplicate. The calculated percentage polydispersities were $8 \%, 5.42 \%$ and $7.77 \%$ and the calculated molecular masses were 97.0, 94.9 and $97.7 \mathrm{kDa}$.

For gel-filtration chromatography, Xcc4156 was diluted to 4, 0.4 and $0.04 \mathrm{mg} \mathrm{ml}^{-1}$ using $50 \mathrm{mM}$ Tris, $150 \mathrm{mM} \mathrm{NaCl} \mathrm{pH} 8$. The same buffer was used for isocratic elution. $100 \mu \mathrm{l}$ protein solution was applied onto a Superdex 200 10/300 column with a bed volume of $\sim 21 \mathrm{ml}$. The molecular weight was obtained following calibration with ferritin $(440 \mathrm{kDa})$, conalbumin (75 kDa), ovalbumin (44 kDa) and RNase A (13.7 kDa). Peak detection was performed in UNICORN (version 5.20). The obtained molecular masses were 105,105 and $102 \mathrm{kDa}$ for the samples at 4, 0.4 and $0.04 \mathrm{mg} \mathrm{ml}^{-1}$, respectively.

\subsection{Protein crystallization and soaking}

For crystallization of the apo protein, Xcc4156 was diluted to $10 \mathrm{mg} \mathrm{ml}^{-1}$ with $10 \mathrm{~m} M$ Tris, $150 \mathrm{mM} \mathrm{NaCl} \mathrm{pH} 8$. Crystallization conditions were screened using The JCSG Core IV Suite (Qiagen, Germany) in MRC 2 Lens Plates (SWISSCI, Switzerland). A bunch of thin needles appeared within one day at $20^{\circ} \mathrm{C}$ in $1 M$ sodium/potassium tartrate, $0.1 M \mathrm{MES} \mathrm{pH}$
6.0 using a drop size of $0.3 \mu \mathrm{l}$ and a protein:reservoir solution ratio of 2:1. Optimization ( $0.6 M$ sodium/potassium tartrate, $0.1 M$ MES pH 6.5; drop volume $3 \mu \mathrm{l}$ ) yielded thicker needles, but the crystals remained intergrown. Prior to data collection, the protruding part of a large needle was briefly transferred into a solution consisting of $0.7 \mathrm{M}$ sodium/potassium tartrate, $0.1 M$ MES pH 6.5, 17.5\% L-2,3-butanediol and flash-cooled in liquid nitrogen.

For soaking, another optimization plate $(0.6 \mathrm{M}$ sodium/ potassium tartrate, 0.1 $M$ MES pH 6.0) was set up with a drop size of $1.5 \mu \mathrm{l}$ containing protein and reservoir solution in the same ratio as before. When the crystals had grown (usually within a week), an additional $0.5 \mu \mathrm{l}$ of ligand solution ['FAD' (0.6 $M$ sodium/potassium tartrate, $0.1 M$ MES $\mathrm{pH}$ 6.0, $15 \mathrm{~m} M$ FAD), 'NaBr' (0.6 $M$ sodium/potassium tartrate, 0.1 $M$ MES $\mathrm{pH}$ 6.0, $150 \mathrm{~m} M \mathrm{NaBr})$, 'FAD-NaBr' $(0.6 M$ sodium/potassium tartrate, $0.1 M$ MES pH 6.0, $15 \mathrm{~m} M$ FAD, $150 \mathrm{~m} M \mathrm{NaBr}$ ) or ' $\mathrm{NaBr} 200$ ' $(0.6 \mathrm{M}$ sodium/potassium tartrate, $0.1 \mathrm{M}$ MES $\mathrm{pH}$ 6.0, $200 \mathrm{~m} M \mathrm{NaBr})]$ was added to the drop. Incubation was performed for one day. Photographs were taken after 0 and $24 \mathrm{~h}$. Crystals were harvested after $24 \mathrm{~h}$ of soaking following the protocol used for the apo crystals but with the ligands present in the cryosolution at the same concentration as in the ligand solution. 
Table 2

List of halogenases structurally compared with Xcc4156 chain $A$.

The colours are in accordance with Fig. 3, grouping the halogenases that belong to the C5 Trp halogenase subgroup (red), the C7 Trp halogenase subgroup (green), the Xcc4156/BrvH subgroup (blue) and an 'outgroup' of other halogenases that are less structurally related to Xcc4156 (grey; refer to Supplementary Fig. S1 for a phylogenetic tree). The description gives an idea of the halogenase family that each listed halogenase belongs to. Variant B halogenases act on carrier proteintethered substrates. The sequence identities output by Clustal Omega were obtained from sequence-based alignment. The structural similarity was output by the $D A L I$ 'all-against-all structure comparison' as a similarity matrix. For halogenases where multiple structures have been published or multiple chains were present in the asymmetric unit, the PDB code and chain were used that had the highest similarity to Xcc 4156 chain $A$. The ligands present in the structure were mostly the cofactor FAD and halide $\mathrm{Cl}$ and, in the case of PrnA, the halogenation product 7-chlorotryptophan. Ligands in italics are presumably irrelevant and are only present owing to the crystallization or cryoprotection conditions. CL, chloride; CTE, 7-chlorotryptophan; EDO, 1,2-ethanediol; FAD, flavin adenine dinucleotide; GOL, glycerol; MES, 2-( $N$-morpholino)-ethanesulfonic acid; PO4, phosphate ion; SO4, sulfate ion; TLA, L-(+)-tartaric acid.

\begin{tabular}{|c|c|c|c|c|c|c|}
\hline Name & Description & $\begin{array}{l}\text { Sequence identity } \\
\text { (Clustal Omega) }(\%)\end{array}$ & $\begin{array}{l}\text { Structural similarity } \\
\text { score }(D A L I)\end{array}$ & $\begin{array}{l}\text { PDB code and } \\
\text { chain ID }\end{array}$ & Ligands in chain & Reference \\
\hline $\mathrm{BrvH}$ & Indole halogenase & 45.5 & 50.4 & 6frl chain $A$ & $T L A, M E S, G O L$ & Neubauer et al. (2018) \\
\hline $\mathrm{PyrH}$ & Trp-5 halogenase & 33.7 & 46.8 & 2wes chain $A$ & FAD, CL & Zhu et al. (2009) \\
\hline Tar14 & Trp-6 halogenase & 32.0 & 45.0 & 6nsd chain $B$ & FAD, $\mathrm{SO}_{4}$ & Luhavaya et al. (2019) \\
\hline $\operatorname{Prn} A$ & Trp-7 halogenase & 32.3 & 44.8 & 2ar8 chain $A$ & FAD, CL, CTE & Dong et al. (2005) \\
\hline Thal & Trp-6 halogenase & 32.6 & 44.4 & 6 h43 chain $B$ & PO4, GOL & Moritzer et al. (2019) \\
\hline Th-Hal & Trp-6 halogenase & 33.7 & 43.9 & 5lv9 chain $B$ & - & Menon et al. (2016) \\
\hline Mpy16 & Pyrrole halogenase (variant B) & 18.1 & 28.1 & 5buk chain $A$ & FAD, $G O L$ & El Gamal et al. (2016) \\
\hline $\mathrm{CndH}$ & Phenol halogenase (variant B) & 16.8 & 27.4 & 3e1t chain $A$ & FAD, CL & Buedenbender et al. (2009) \\
\hline Bmp2 & Pyrrole halogenase (variant B) & 15.3 & 26.7 & 5bva chain $A$ & FAD, $E D O$ & El Gamal et al. (2016) \\
\hline
\end{tabular}

\subsection{Protein structure determination}

Measurements were carried out on the BL14.2 beamline at Helmholtz-Zentrum Berlin (Gerlach et al., 2016). For both apo and soaked Xcc4156 crystals, 3600 frames were collected with an oscillation angle of $0.1^{\circ}$. The $\mathrm{X}$-ray wavelength was $0.917143 \AA$ for the apo crystals and $0.9184 \AA$ for the soaked crystals. The raw data were processed with $X D S$ and scaled with XSCALE (Kabsch, 2010). For the apo data set, the data were imported into $C C P 4(C C P 4 i 2$ interface; Potterton et al., 2018) and merged [cut at $I / \sigma(I)=2$ ] with AIMLESS (Evans \& Murshudov, 2013) for structure solution via molecular replacement using MrBUMP (Keegan \& Winn, 2007; McCoy et al., 2007); the best search model was chain $A$ of BrvH (PDB entry $6 \mathrm{frl}$ ), with a sequence identity of $45.5 \%$. An initial model was built by Buccaneer (Cowtan, 2006). Manual model building was performed in Coot (Emsley et al., 2010), and both REFMAC5 (Murshudov et al., 2011) and phenix.refine (Liebschner et al., 2019) were used for crystallographic refinement of the interim models. Restrained refinement was used and $B$ factors were considered to be isotropic. Noncrystallographic symmetry (NCS) was used to generate further restraints. Validation with MolProbity (Chen et al., 2010) was part of the building process. The statistics of the final model are given in Table 1, and the coordinates and structure factors have been deposited in the Protein Data Bank (PDB entry $6 y 1 w)$.

Data processing of data sets from the soaked crystals was performed similarly to the apo data set, but as we were looking for anomalous signal, Friedel mates were treated as different reflections for soaks containing $\mathrm{NaBr}$. As the soaked and apo crystals had very similar unit-cell parameters, rigid-body refinement of the apo model against the respective data followed by three cycles of restrained refinement was performed in phenix.refine. The apo model was used to generate additional restraints. The resulting electron-density maps were evaluated in Coot with a focus on the FAD-binding and halide-binding sites. No further model building was performed, and the structures were not deposited in the PDB. The statistics of the models are given in Table 1. Figures were generated in $P Y M O L$.

\subsection{Bioinformatics}

A multiple sequence alignment was performed in Clustal Omega (Madeira et al., 2019) using the default settings. 44 FDH sequences (given in the supporting information) were input and a phylogenetic tree was calculated from the resulting alignment. The tree was calculated in Jalview 2.10.5 (Waterhouse et al., 2009) using the neighbour-joining algorithm and the BLOSUM62 matrix. The structures of the most similar halogenases (Table 2) and halogenases that clustered more distantly with the Trp halogenases were additionally compared with the Xcc4156 structure using the DALI server (Holm, 2019) in an 'all-against-all structure comparison'. The resulting comparison was uploaded to ClustalW2 (Madeira et al., 2019) in order to generate a phylogenetic tree using the neighbour-joining algorithm and distance correction including gaps.

In addition, a DALI comparison (Holm, 2019) of the structures of Xcc4156 (PDB entry 6y1w chain $A$ ), BrvH (PDB entry 6 frl chain $A$; Neubauer et al., 2018), Th-Hal (PDB entry 5lv9 chain $B$; Menon et al., 2016) and RebH (PDB entry 2oal chain B; Yeh et al., 2007) was performed and used as input for the ESPript 3.0 server (Robert \& Gouet, 2014). The alignment was also used in Fig. 2, in which the conservation of the amino acids at each position is shown. This quantitative annotation was output by Jalview (Waterhouse et al., 2009) based on the 
number of conserved physicochemical properties. Its calculation is based on that used in the $A M A S$ method of multiple sequence alignment analysis (Livingstone \& Barton, 1993).

In order to compare the crystal contacts that the loops of RebH (PDB entry 2oam; Yeh et al., 2007) and PltM (PDB entry 6bzn; Mori et al., 2019) as well as Xcc4156 are involved in, the PISA server (Krissinel \& Henrick, 2007) was used.

\section{Results}

\subsection{Crystal structure of Xcc4156}

Our attempts to co-crystallize Xcc4156 with FAD and bromide failed. A total of 2896 -well plates were set up with different commercial screens, at different protein concentrations and at different temperatures (see Supplementary Table S2). No diffracting crystals were obtained. However, apo Xcc4156 yielded crystals that diffracted to a resolution of 1.6 A (see Table 1).

In the crystal, there are two chains in the asymmetric unit, with only minor differences between them (Fig. 1a). Each of these is a halogenase monomer consisting of two subdomains: a 'box' subdomain containing the FAD-binding site, which is structurally conserved among FDHs, and a more variable 'pyramid' subdomain (Fig. 1b). The substrate-binding site of FDHs is at the interface between the box and pyramid subdomains (Dong et al., 2005). The dimer observed in the crystal is very similar to the crystallographic dimers of Trp halogenases (Dong et al., 2005; Yeh et al., 2007; Zhu et al., 2009; Shepherd et al., 2016; Menon et al., 2016; Moritzer et al., 2019; Luhavaya et al., 2019; Lingkon \& Bellizzi, 2020). Estimates of the molecular weight from the hydrodynamic radius obtained by size-exclusion chromatography (SEC) and dynamic light scattering (DLS) are 105 and $96.5 \pm 1.5 \mathrm{kDa}$, respectively. These values are substantially higher than that of a monomer $(58.1 \mathrm{kDa})$, but are still lower than the molecular weight of a dimer $(116.2 \mathrm{kDa})$. SEC was performed on samples between 4 and $0.04 \mathrm{mg} \mathrm{ml}^{-1}$, giving very similar results. This renders a concentration-dependent change in the oligomerization state very unlikely. As a nonglobular shape can explain an apparent molecular weight that is higher but not lower than the actual value, we take the SEC and DLS results as an indication that Xcc4156 may be monomeric in solution. In Thal, a tryptophan halogenase, SEC and DLS also hinted towards a monomer, as is the case here, but the enzyme likewise formed a dimer in the crystal (Moritzer et al., 2019). ESI-MS under native conditions revealed that Thal was present both as a homodimer and a monomer in solution (Minges et al., 2020). The same might be the case for Xcc4156. The presence of the same dimer in all Trp halogenase crystals, and in solution in the case of PrnA as observed by SEC (Dong et al., 2005), suggests an evolutionary advantage of this association, which is supported by a study on Thal mutants in which increased thermostability coincided with a higher proportion of the protein being present as a homodimer in solution (Minges et al., 2020).

To further investigate the similarity to known halogenases, a multiple sequence alignment of $44 \mathrm{FDH}$ sequences was performed in Clustal Omega (Madeira et al., 2019). Additionally, the Xcc4156 structure was compared with the structures of selected halogenases (Table 2) using the DALI server (Holm, 2019) and a phylogenetic tree was created from the $D A L I$ comparison (Supplementary Fig. S1).

The sequence alignment and structural comparison performed in this study confirmed the high similarity of Xcc4156 to Trp halogenases that we expected based on previous sequence alignments (Ismail et al., 2019). In accordance, Xcc4156 shares their overall structure (see above).

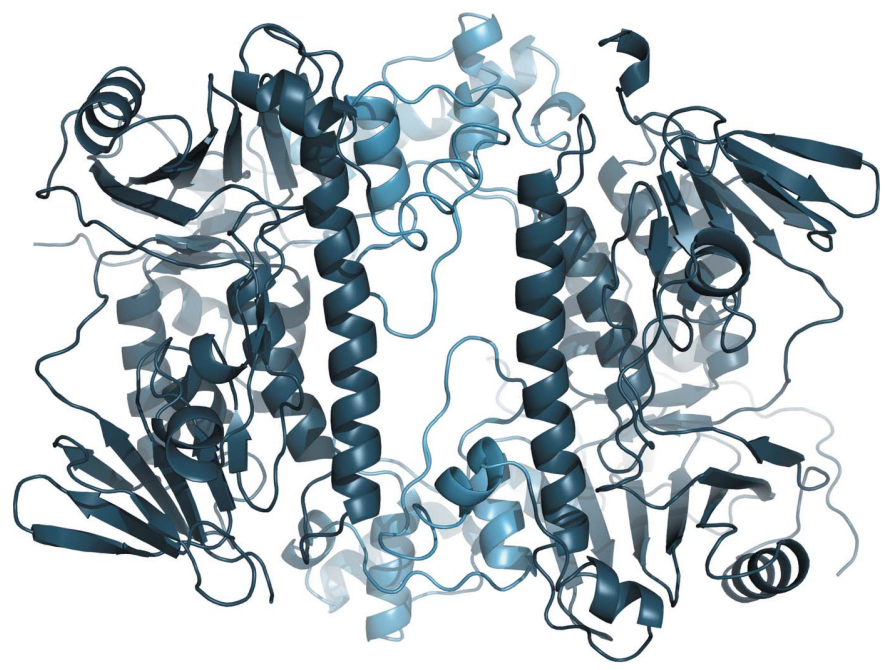

(a)

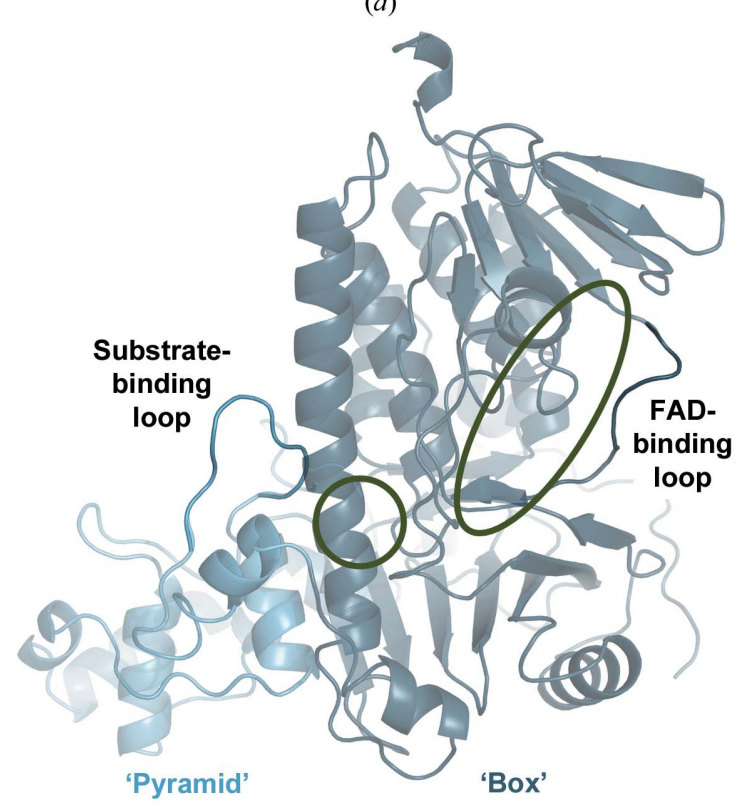

(b)

Figure 1

Crystal structure of Xcc4156. (a) Dimer in the crystal of Xcc4156 (PDB entry 6y1w). The structurally conserved part between different FDHs ('box') is shown in dark blue and the structurally variable 'pyramid' is shown in light blue. $(b)$ Monomer of Xcc4156 (chain $A$ ). The FADbinding loop in the 'box' subdomain and a loop at the substrate-binding site at the interface between the 'box' and the 'pyramid' subdomains are highlighted. The inferred FAD-binding and substrate-binding sites (deduced from their respective positions in Trp halogenases) are marked as green ovals. 
Xcc4156 has $45 \%$ sequence identity to BrvH (Neubauer et al., 2018) and around $30-35 \%$ sequence identity to various Trp halogenases (for example $34 \%$ to both PyrH and RebH and $32 \%$ to $\operatorname{PrnA}$ ).

As expected, based on the high sequence identity, the structure of Xcc4156 resembles that of BrvH (Figs. $2 a$ and $2 b$ ). Their substrate-binding region distinguishes Xcc4156 and $\mathrm{BrvH}$ from the Trp halogenases. When clustered after a $B L A S T$ pairwise alignment, the latter form two groups (Fisher et al., 2019). In addition to other minor differences, particularly in the loops, the substrate coordination differs between these groups (Figs. $2 a-2 d$; Luhavaya et al., 2019). One group, containing, for example, SttH, Th-Hal, PyrH, KtzR and Tar14, has a long substrate-binding loop (' 1 ' in Fig. $2 c$ ). This group includes C5 Trp halogenases, which halogenate their substrate tryptophan in the C5 position, as well as some C6 Trp halogenases. The substrate-binding loop of the second group, which contains, for example, RebH, Thal, PrnA and KtzQ, is shorter and connects to a different side of the pyramid (' 2 ' in
Fig. 2d). This group comprises all known C7 Trp halogenases as well as some C6 Trp halogenases.

Xcc4156 and BrvH have short loops in both positions, resulting in a more open substrate-binding site compared with Trp halogenases and a solvent-accessible catalytic lysine residue. In Trp halogenases the loop usually closes over the substrate upon binding. As Xcc4156 and BrvH have much shorter loops, this seems very unlikely in these two enzymes. The absence of the substrate-binding loop is reflected in the residues adjacent to the substrate-binding site. Whereas the catalytic residues in $\mathrm{RebH}$ all have corresponding and similar residues in Xcc4156 (refer to Supplementary Table S1) and a part of the $\pi$-stacking region is also similar, the backbone binding region of $\mathrm{RebH}$ does not have a counterpart in Xcc4156. Like BrvH, Xcc4156 does not halogenate free tryptophan (Neubauer et al., 2018; Ismail et al., 2019); these enzymes do, however, show activity towards free indole and indole derivatives. This can be explained by the lack of stabilization of tryptophan in the substrate-binding site. Thus,

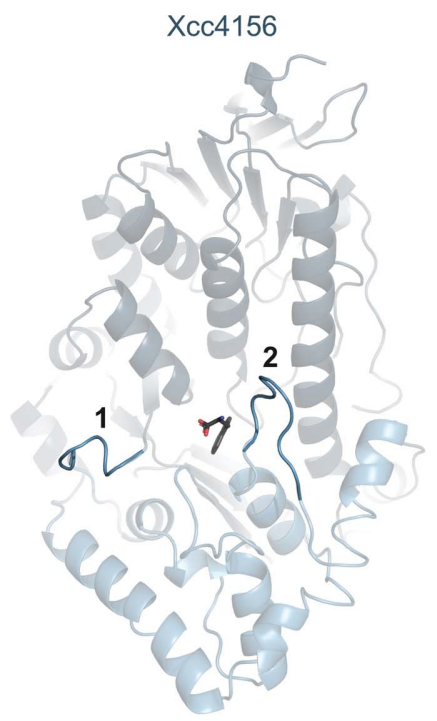

(a)

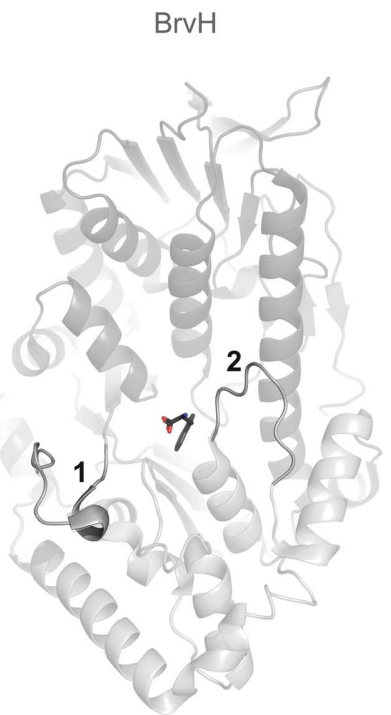

(b)

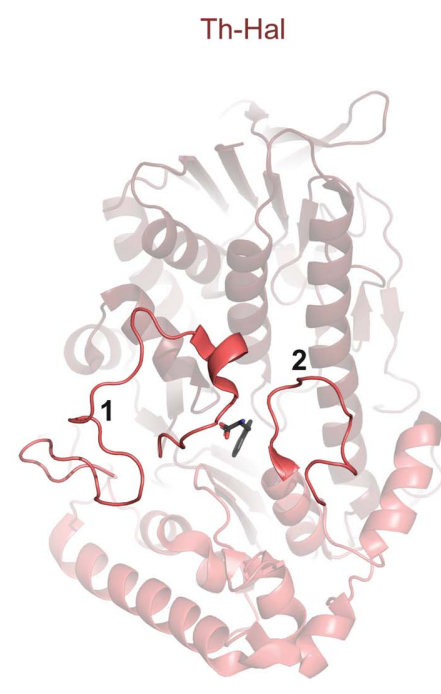

(c)

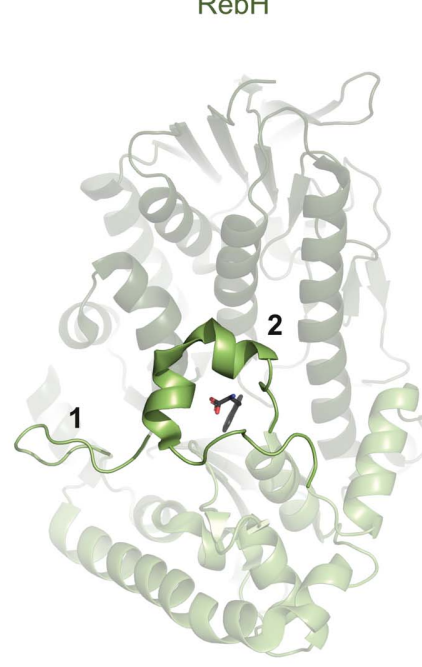

$(d)$

Loop region 1

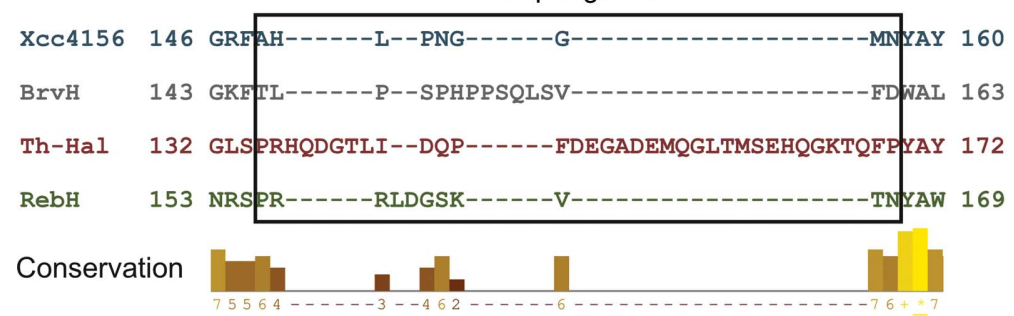

(e)

Figure 2

Structural comparison of Xcc4156 with BrvH, Th-Hal and RebH. (a) Xcc4156 chain $A$ (blue). (b) BrvH (grey; PDB entry 6frl chain $A$ ). (c) Th-Hal (red; PDB entry 5lv9 chain $B) .(d)$ RebH (green; PDB entry 2oa1 chain $A)$. In $(a)-(d)$ the tryptophan from RebH (grey) is shown to mark the location of the substrate-binding site, as neither the Xcc4156 structure nor the BrvH and Th-Hal structures contain a bound substrate. Two loop regions are highlighted that form part of the substrate-binding site in two clades of halogenases. Region ' 1 ' is the substrate-binding loop of the C5 Trp halogenase group containing Th-Hal (amino acids 149-157, 146-160, 135-169 and 156-166 in Xcc4156, BrvH, Th-Hal and RebH, respectively). Region '2' is the substratebinding loop of the C7 Trp halogenase group that RebH belongs to (amino acids 428-438, 434-444, 443-457 and 442-467 in Xcc4156, BrvH, Th-Hal and $\mathrm{RebH}$, respectively). Xcc4156 and BrvH have a short loop in both positions, resulting in a very open substrate-binding site. Refer to Supplementary Fig. $\mathrm{S} 1$ for a phylogenetic tree. (e) Alignment based on a DALI structure comparison showing the sequences of Xcc4156, BrvH, Th-Hal and RebH in both loop regions. The rectangle encloses the loop regions that are highlighted in $(a)-(d)$. The conservation of the amino acids (yellow) was output by Jalview. 
the hypothesis that $\mathrm{BrvH}$ might halogenate a peptide (Neubauer et al., 2018), like MibH (Ortega et al., 2017), also applies to Xcc4156. A peptide or larger tryptophan derivative could interact with the halogenase surface, orienting the substrate in the active site. It should be noted, however, that Xcc4156 only shares $26 \%$ sequence identity with MibH, and both Xcc4156 and BrvH do not cluster close to MibH based on sequence alignment (data not shown), an observation that was also made by Fisher et al. (2019).

The FAD-binding loop (amino acids 47-56) of Xcc4156 does not have continuous electron density in chain $B$ (Supplementary Fig. S4b). Its position seems to be mainly stabilized by a weak interaction between the carbonyl $\mathrm{O}$ atom of Gly51 and a hydrogen of the guanidino group of Arg313 (both in chain $B$ ). In chain $A$, however, the loop is well defined as it is part of a crystal contact that is stabilized by two hydrogen bonds between the backbone $\mathrm{N}$ and $\mathrm{O}$ atoms of Val53 in chain $A$ and the side-chain amide of Gln470 in chain $B$ (Figs. $3 a$ and S4a). The FAD-binding loop of FDHs has been hypothesized to adopt two conformations. In apo crystals of Thal the FAD-binding loop adopts an 'open' conformation (Fig. 3c), whereas in FAD-bound structures the loop closes (Fig. 3d; Moritzer \& Niemann, 2019). The side chain of a glutamate flanking the FAD-binding loop flips by almost $180^{\circ}$ upon FAD binding in Thal (Figs. $3 c$ and $3 d$ ) and PyrH (Figs. $3 e$ and $3 f$ ). Although the loop of apo Xcc4156 would be expected to resemble that of apo Thal, it has similarities to both the open and closed states (Fig. 3g). However, Glu57 in Xcc4156 adopts the same conformation as Glu49 in apo Thal and apo PyrH, indicating that the loop is in its open conformation (Figs. $3 a, 3 c$ and $3 e$ ). While part of the loop differs between chain $A$ and $B$ in apo Xcc4156 (Fig. $3 b$ ), probably owing to the crystal contact in chain $A$, Glu57 has the same orientation in both chains.

Instead of FAD, the FAD-binding site in both chains of Xcc4156 contains a well defined L-tartrate ion (Fig. $3 h$ and Supplementary Figs. S2a-S2c) which does not directly interact with the loop. Tartrate was used as the precipitant in

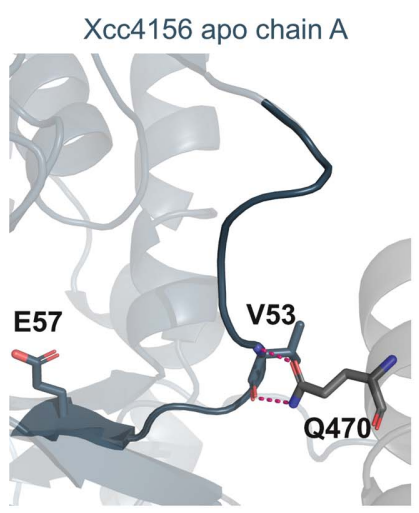

(a)

$$
\text { PyrH apo }
$$

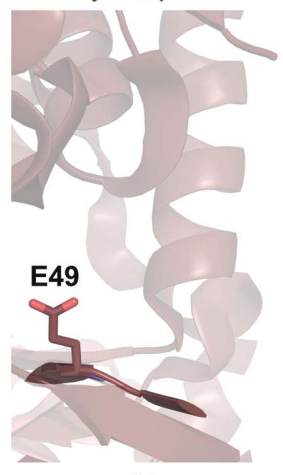

(e)

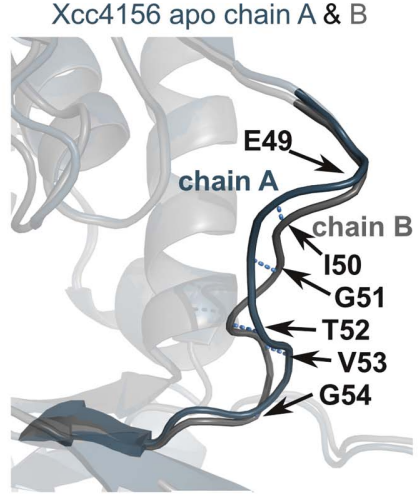

(b)

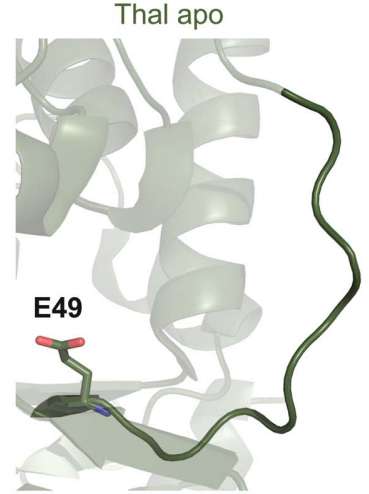

(c) $\mathrm{PyrH}+\mathrm{FAD}$

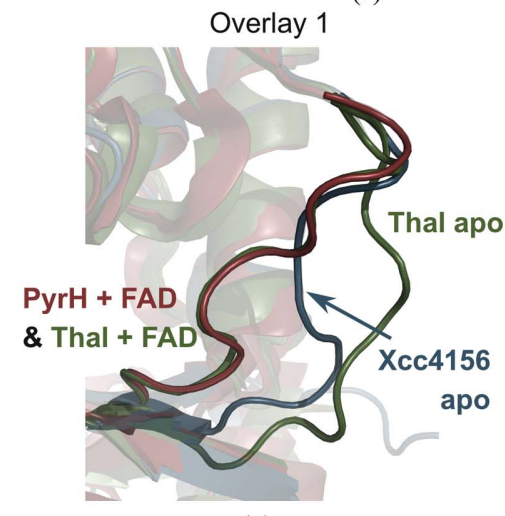

$(g)$

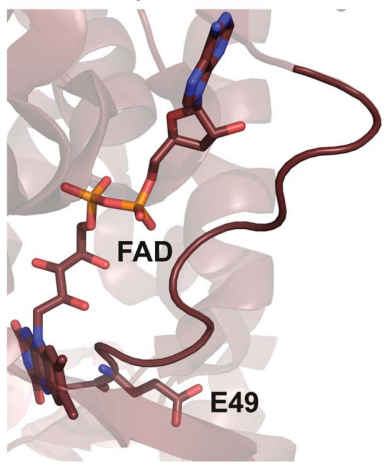

$(f)$
Figure 3

Comparison of the FAD-binding loop of Xcc4156 with the open and closed FAD-binding loops of Thal and PyrH. (a) The crystal contact that fixes the loop in chain $A$ (blue; amino acids 47-58) of Xcc4156 in position. The backbone atoms of Val53 in chain $A$ form two hydrogen bonds (pink) to the Gln 470 side-chain amide in chain $B$ of a symmetry-related molecule (grey). (b) Overlay of chain $A$ of Xcc4156 (blue) with chain $B$ (grey). Light blue dashed lines connect the same amino acids in both chains. The distances between $\mathrm{C}^{\alpha}$ atoms are 0.8, 1.7, 2.5, 2.9, 2.6 and 1.0 $\AA$ for Glu49-Gly54. (c) The FAD-binding loop of Thal (green; PDB entry $6 \mathrm{~h} 43$ chain $A$; amino acids 39-50) in the 'open' conformation when no FAD is bound. (d) Upon FAD binding, the FADbinding loop closes (PDB entry 6sls chain $A$ ). (e) In the absence of FAD, the FAD-binding loop of PyrH could not be modelled (red; PDB entry 2 weu; amino acids 36-47). $(f)$ When FAD is bound the loop closes, taking on the same conformation as in Thal (PDB entry 2 wet chain $A)$. In $(a),(c),(d),(e)$ and $(f)$, a conserved glutamate residue (Glu57 in Xcc4156 and Glu49 in both Thal and PyrH) is shown which acts as a sensor for FAD binding. ( $g$ ) Overlay of apo Xcc4156 chain $A$ (blue), apo Thal (green), Thal + FAD (green) and PyrH + FAD (red). Loops are open in the apo state and closed in the FAD-bound state. ( $h$ ) Overlay of apo Xcc4156 chain $A$ (blue) and Thal + FAD (green). In Xcc4156, an L-tartaric acid (TLA) molecule is bound at the site that coordinates a phosphate of FAD in other halogenases. 
crystallization. Interestingly, the same tartrate-binding site can be observed in two other halogenases: an unnamed halogenase and BrvH (PDB entries 2pyx and 6frl; Joint Center for Structural Genomics, unpublished work; Neubauer et al., 2018; Supplementary Figs. S2 $d$ and S2e). The coordination is the same in all three cases, with most adjacent water molecules at the same positions and the involvement of three backbone $\mathrm{N}$
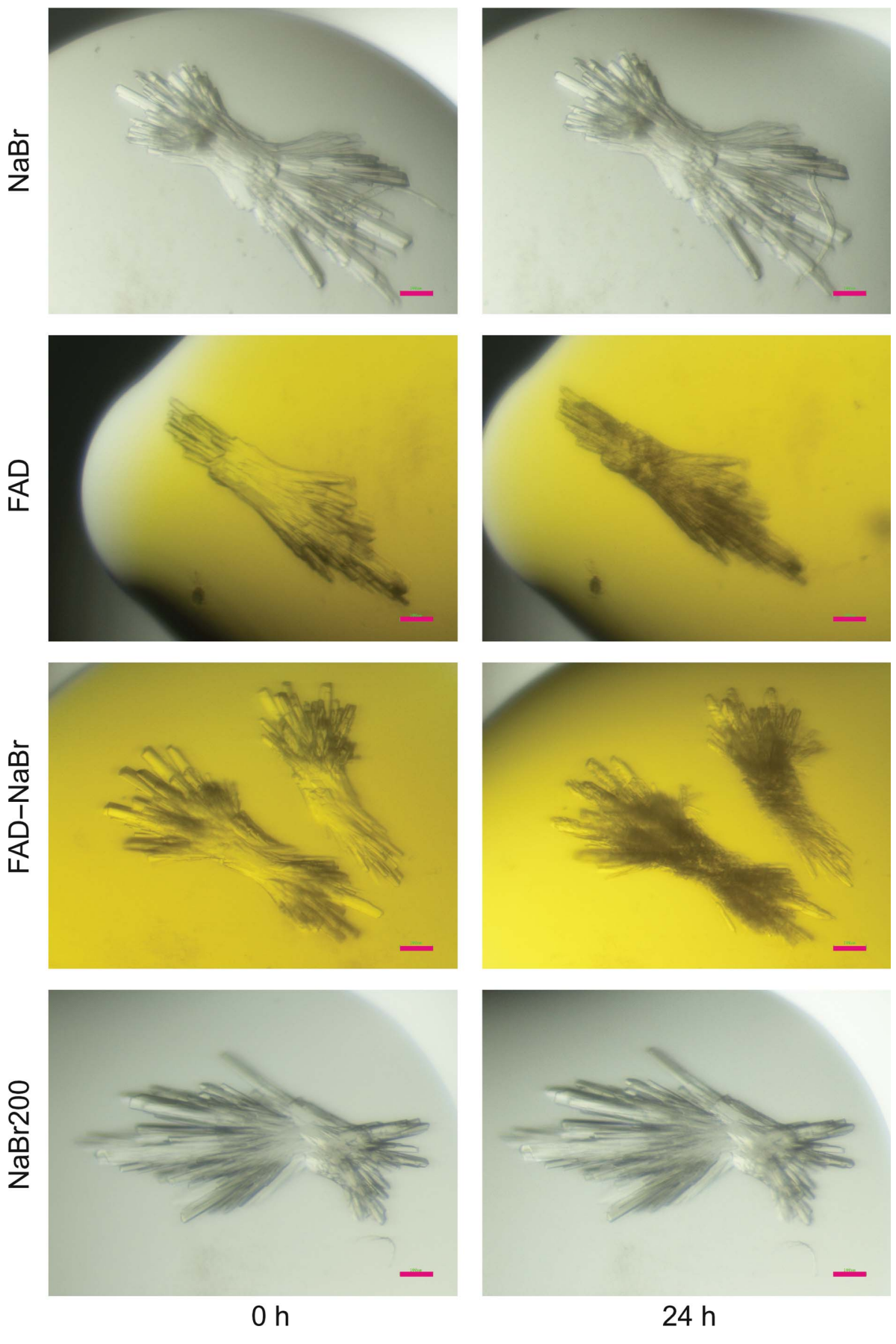

Figure 4

Soaking of apo crystals of Xcc4156. Soaks were performed by the addition of $0.5 \mu 1150 \mathrm{~m} M \mathrm{NaBr}$ ('NaBr'), $15 \mathrm{~m} M$ FAD ('FAD'), $15 \mathrm{~m} M$ FAD and $150 \mathrm{~m} M$ NaBr ('FAD-NaBr') or $200 \mathrm{~m} M \mathrm{NaBr}$ ('NaBr200') in reservoir solution to a drop consisting of $0.5 \mu \mathrm{l}$ reservoir solution and $1 \mu \mathrm{l}$ protein solution. Photographs were taken directly after the addition of the ligand solution to the drop (' $0 \mathrm{~h}$ ') and after $24 \mathrm{~h}$. The pink bar indicates $100 \mu \mathrm{m}$. atoms (Thr23, Ala24 and Leu335 in Xcc4156; Thr16, Ala17 and selenomethionine Met351 in PDB entry 2pyx; and Thr19, Ala20 and Leu340 in BrvH). Two corresponding residues (Ala16 and Leu349) in Thal coordinate one phosphate of the FAD (PDB entry 6sls; Supplementary Fig. S2f).

Another striking feature of the FAD-binding site is the side chain of Asn347 (Supplementary Fig. S3). This amino acid is the second amino acid of the canonical halide-binding site that has been observed in FDHs (Supplementary Fig. S5). The latter site is close to the isoalloxazine ring of the FAD and consists of two backbone NH groups that coordinate the halide. Whether this site is relevant for halogenation or is only an artefact of crystallization remains unclear (Blasiak \& Drennan, 2009). In apo Xcc4156, this site (Thr346 and Asn347) does not contain any bound halide. In most FDHs, a glycine occupies the second position of this conserved region. This led Gkotsi et al. (2019) to postulate the halogenaserecognition sequence motif $\mathrm{F} x$.P $x . \mathrm{S} x . \mathrm{G}$ (where ' $x$.' denotes any number of amino acids) in addition to the established $\mathrm{G} x \mathrm{G} x x \mathrm{G}$ and $\mathrm{W} x \mathrm{~W} x \mathrm{IP}$ motifs. The glycine residue in their proposed sequence motif is conserved in almost all known FDHs. Exceptions are BrvH and the other two $X$. campestris halogenases characterized by the Sewald laboratory (Neubauer et al., 2018; Ismail et al., 2019), which all have a serine at this position. The asparagine in Xcc4156 is particularly interesting as its side chain occupies the position that would be taken up by the ribityl moiety of FAD in a FAD-bound halogenase, thus possibly hampering FAD binding (Supplementary Figs. S3 $a$ and S $3 b$ ). The amide $\mathrm{N}$ atom is within $1 \AA$ of the $\mathrm{C}_{2}^{\prime}$ atom of a FAD molecule from Thal when superimposed with Xcc4156.

\subsection{Soaking of apo Xcc4156 crystals}

After unsuccessfully attempting to cocrystallize Xcc4156 with its cofactor FAD and $\mathrm{NaBr}$ (Supplementary Table S2), soaking of apo crystals was performed. Thin, intergrown needles grew within one or a few days of setting up the plates. For soaking, reservoir solution containing the respective ligand(s) was added to the drop (Fig. 4). 
When $150 \mathrm{~m} M \mathrm{NaBr}$ was added, the crystals were not harmed and retained their appearance. The addition of $15 \mathrm{mM}$ FAD led to a visual deterioration of the crystals, and the addition of both $15 \mathrm{~m} M$ FAD and $150 \mathrm{~m} M \mathrm{NaBr}$ led to a worse crumbling of the crystals. To verify that this effect was not caused by the higher ionic strength of the latter solution, crystals were soaked with $200 \mathrm{~m} M \mathrm{NaBr}$. These crystals, like those at a lower $\mathrm{NaBr}$ concentration, did not show any sign of harm. Interestingly, the crumbling of the crystals appeared to be much worse for smaller crystals and intergrown areas, suggesting that single large crystals are less prone to deterioration.

\subsection{Crystal structures of soaked Xcc4156}

The soaked crystals were harvested and data were collected (see Table 1 for data statistics). The structures were solved by refinement of the final apo model against the respective data. For crystals soaked with $\mathrm{NaBr}$ no bromide could be observed in the halide-binding site despite the presence of bromide in other sites as confirmed by an anomalous difference map (Fig. 5).

When data sets were collected from crystals soaked with FAD (or FAD and $\mathrm{NaBr}$ ), we noticed that around half of the crystals showed little to no diffraction (to less than $4 \AA$ resolution, compared with around $2-3 \AA$ for apo crystals or $\mathrm{NaBr}$ soaks). Interestingly, in all of the remaining data sets (one and three data sets from crystals soaked with FAD or with FAD and $\mathrm{NaBr}$, respectively) the FAD-binding sites were empty save for tartrate, which was present at the same site as in the apo crystals in both chains. The crystals soaked with FAD thus showed similar electron density to the apo crystals, whereas those soaked with both FAD and $\mathrm{NaBr}$ resembled the $\mathrm{NaBr}$-soaked crystals. As the soaked structures did not reveal any relevant structural information

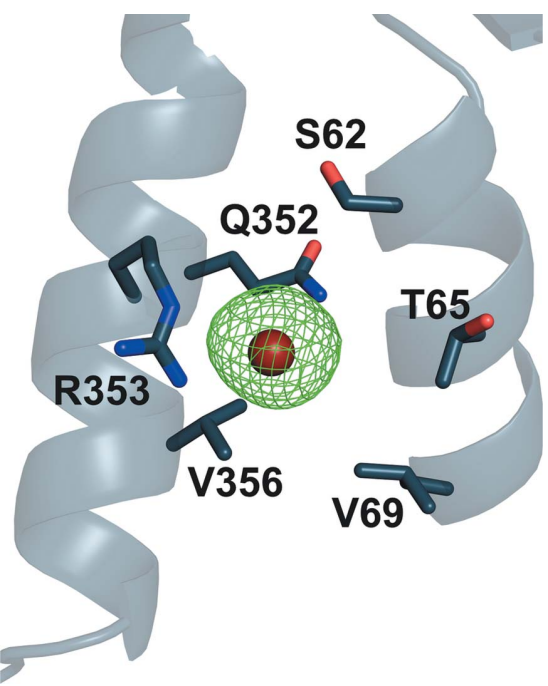

Figure 5

Anomalous difference density of a bromide ion in an Xcc4156 crystal soaked with $\mathrm{NaBr}$. The bromide ion is coloured brown; the anomalous difference density (green mesh) is contoured at $3 \sigma$. Side chains at a distance of less than $6 \AA$ from the bromide are shown as sticks. beyond the apo structure and had considerably lower resolution, we did not perform further model building or deposit these structures in the PDB.

The visual deterioration of the crystals, the partial loss of diffraction and the finding that no electron density for FAD was present upon soaking suggest that binding of FAD might destroy the crystals. When no FAD is bound the crystals still diffract, but the resulting electron density shows no sign of $\mathrm{FAD}$, and when FAD is bound the diffraction is gravely reduced.

\section{Discussion}

We observed a visual deterioration of apo crystals of the flavin-dependent halogenase Xcc4156 upon the addition of FAD, which was even more dramatic when bromide was present as well. This might be explained by the binding of FAD causing a change in the position of the FAD-binding loop. Indeed, the loop is partly disordered in one chain of the apo structure and is fixed in an open conformation in the other chain.

There are four other halogenases, RebH, PyrH, PltM and Thal, for which both apo and holo structures are available. In apo PyrH, the FAD-binding loop (amino acids 37-43) is disordered and was not modelled (Zhu et al., 2009). In the presence of FAD the loop adopts an ordered 'closed' conformation. The loop is close to the FAD and interacts with it through backbone atoms. It should be mentioned that the apo and FAD-bound structures of $\mathrm{PyrH}$ have different space groups and crystal packing, the reasons for which are unclear as the crystals grew under the same conditions. Thal behaves similarly to $\mathrm{PyrH}$, with the exception that in the apo form it exhibits a well defined loop in the 'open' conformation, leaving space between the loop and the potential FAD. The loop closes when Thal is soaked with FAD (Moritzer \& Niemann, 2019). In both RebH and PltM the FAD-binding loop, as in Thal, is well defined in the apo state; however, the loop position does not change in the presence of the cofactor. This can be explained as in both structures the FAD-binding loop is part of a crystal contact, with the loops of two chains interacting with each other, generating a noncrystallographic twofold axis. In PltM, the conformation is very similar to that observed for 'closed' loop conformations (Mori et al., 2019). This is in contrast to the 'open' loop position in RebH (Bitto et al., 2008), where the loop is not in direct contact with the cofactor, leaving the FAD-binding site solvent exposed. Unlike in RebH and PltM, soaking Xcc4156 crystals with FAD seems to have caused the FAD-binding loop to change its position, resulting in visible harm to the crystals.

In order to explain this different behaviour, the crystal contacts that the loops are involved in were compared using the PISA server (Krissinel \& Henrick, 2007). In Xcc4156 only four residues are part of the relevant interface, as opposed to six or five residues in apo RebH and apo PltM, respectively. The average buried area of the residues within the loop is $55 \%$ for $\mathrm{RebH}, 42 \%$ or $54 \%$ for PltM (with four monomers in the 
asymmetric unit; thus, two dimers that interact via their loops) and only $27.5 \%$ for Xcc4156. This might indicate a weaker interaction that can be disrupted as FAD binds. Binding of FAD in chain $A$ and the resulting elimination of the crystal contact seems to have led to a loss in diffraction of the crystals. The crystals of Xcc4156 that still showed diffraction had no FAD bound in chain $A$, as would be expected. Interestingly, however, soaking the crystals with FAD also did not result in FAD being bound by the less ordered FAD-binding loop (chain $B$ ) of the crystals. The high concentration of tartrate (600 $\mathrm{m} M$ compared with $15 \mathrm{~m} M$ FAD) and its presence in the FAD-binding site could explain the absence of FAD but not the observed crystal cracking.

We also observed that the visual deterioration of the crystals during soaking was more severe when $\mathrm{NaBr}$ was present in addition to FAD, which could not be explained by the higher ionic strength of the solution. However, the halide binding might have a cooperative effect, promoting the binding of FAD. This would enable the soaking solution to affect the crystals more dramatically. Without FAD, even in the presence of high concentrations of $\mathrm{NaBr}$ no halide is bound in the proposed halide-binding site. This is in accordance with the literature. Out of 50 published structures of FDHs (as of 8 January 2020), 15 have no FAD and no halide bound in the respective binding sites (apo), 16 have only FAD modelled (either because no halide is present or because it was not modelled) and another 12 have both FAD and the halide modelled in at least one chain. Of the seven remaining structures, one PrnA structure (PDB entry 2ard) contains $\mathrm{FADH}_{2}$ with no halide modelled (Dong et al., 2005) and two PltM structures (PDB entries 6bza and 6bzz) contain FAD that is bound in an unusual pose (Mori et al., 2019). One RebH structure (PDB entry 2oa1) contains FAD and chloride in one chain, but only adenine and no halide modelled in the other (Bitto et al., 2008), and one Thal structure (PDB entry 6slt) contains only FAD in one chain, while in the other only AMP was modelled (Moritzer \& Niemann, 2019). The modelling of only parts of the FAD in the latter two cases can be explained as the adenine moiety is bound by a nonflexible region and the riboflavin moiety (which forms part of the halide-binding site) is more flexible in these cases, making its modelling harder or impossible (Moritzer \& Niemann, 2019). The MibH structure (PDB entry 5uao) contains both FAD and chloride in two chains and neither of them in two other chains (Ortega et al., 2017). The PltA structure (PDB entry 5dbj) contains FAD in all five chains, but chloride is only present in four chains (Pang et al., 2015). The correlation between the binding of FAD and the halide strongly implies a dependence of halide binding on FAD binding, as has been proposed by Mori et al. (2019). The cooperative effect that we observed substantiates our hypothesis that FAD binding causes the loop to close and the crystals to break and makes a nonspecific effect of FAD binding unlikely. As bromide soaks on their own do not negatively affect the crystal integrity, it is hard to rationalize the exacerbated damage to crystals upon soaking with FAD and bromide other than through cooperative binding at the evolved halide-binding site formed by the protein and FAD.
We cannot completely rule out that nonspecific binding of bromide and FAD to the protein surface causes this effect, but this appears to us to be highly unlikely.

In summary, we observed the cracking of apo crystals of the halogenase Xcc 4156 upon the addition of FAD to the crystals. This could be explained by a change in the position of the FAD-binding loop, which was part of a crystal contact. This effect was increased in the presence of bromide, suggesting a cooperative effect between the binding of FAD and the halide.

\section{Acknowledgements}

We thank Helmholtz-Zentrum Berlin (HZB) for the allocation of synchrotron-radiation beamtime and the dedicated beamline scientists Dr Christian Feiler and Dr Thomas Hauss.

\section{Funding information}

We gratefully acknowledge the financial support from HZB. The position of $\mathrm{CW}$ was funded by the rectorate of Bielefeld University as a strategic project. MI acknowledges funding from the German Academic Exchange Service (DAAD) and the Bielefelder Nachwuchsfonds of Bielefeld University.

\section{References}

Agarwal, V., El Gamal, A. A., Yamanaka, K., Poth, D., Kersten, R. D., Schorn, M., Allen, E. E. \& Moore, B. S. (2014). Nat. Chem. Biol. 10, 640-647.

Ainsley, J., Mulholland, A. J., Black, G. W., Sparagano, O., Christov, C. Z. \& Karabencheva-Christova, T. G. (2018). ACS Omega, 3, 4847-4859.

Bitto, E., Huang, Y., Bingman, C. A., Singh, S., Thorson, J. S. \& Phillips, G. N. Jr (2008). Proteins, 70, 289-293.

Blasiak, L. C. \& Drennan, C. L. (2009). Acc. Chem. Res. 42, 147-155. Buedenbender, S., Rachid, S., Müller, R. \& Schulz, G. E. (2009). J. Mol. Biol. 385, 520-530.

Chen, V. B., Arendall, W. B., Headd, J. J., Keedy, D. A., Immormino, R. M., Kapral, G. J., Murray, L. W., Richardson, J. S. \& Richardson, D. C. (2010). Acta Cryst. D66, 12-21.

Cowtan, K. (2006). Acta Cryst. D62, 1002-1011.

Domergue, J., Erdmann, D., Fossey-Jouenne, A., Petit, J.-L., Debard, A., de Berardinis, V., Vergne-Vaxelaire, C. \& Zaparucha, A. (2019). $A M B$ Express, 9, 175.

Dong, C., Flecks, S., Unversucht, S., Haupt, C., van Pée, K.-H. \& Naismith, J. H. (2005). Science, 309, 2216-2219.

Dorrestein, P. C., Yeh, E., Garneau-Tsodikova, S., Kelleher, N. L. \& Walsh, C. T. (2005). Proc. Natl Acad. Sci. USA, 102, 13843-13848.

Durak, L. J., Payne, J. T. \& Lewis, J. C. (2016). ACS Catal. 6, 14511454.

El Gamal, A., Agarwal, V., Diethelm, S., Rahman, I., Schorn, M. A., Sneed, J. M., Louie, G. V., Whalen, K. E., Mincer, T. J., Noel, J. P., Paul, V. J. \& Moore, B. S. (2016). Proc. Natl Acad. Sci. USA, 113, 3797-3802.

Emsley, P., Lohkamp, B., Scott, W. G. \& Cowtan, K. (2010). Acta Cryst. D66, 486-501.

Evans, P. R. \& Murshudov, G. N. (2013). Acta Cryst. D69, 1204-1214.

Fisher, B. F., Snodgrass, H. M., Jones, K. A., Andorfer, M. C. \& Lewis, J. C. (2019). ACS Cent. Sci. 5, 1844-1856.

Flecks, S., Patallo, E. P., Zhu, X., Ernyei, A. J., Seifert, G., Schneider, A., Dong, C., Naismith, J. H. \& van Pée, K.-H. (2008). Angew. Chem. Int. Ed. 47, 9533-9536.

Fraley, A. E., Garcia-Borràs, M., Tripathi, A., Khare, D., MercadoMarin, E. V., Tran, H., Dan, Q., Webb, G. P., Watts, K. R., Crews, P., 
Sarpong, R., Williams, R. M., Smith, J. L., Houk, K. N. \& Sherman, D. H. (2017). J. Am. Chem. Soc. 139, 12060-12068.

Frese, M., Schnepel, C., Minges, H., Voss, H., Feiner, R. \& Sewald, N. (2016). Chem CatChem, 8, 1799-1803.

Gerlach, M., Mueller, U. \& Weiss, M. S. (2016). J. Large-Scale Res. Facil. 2, A47.

Gkotsi, D. S., Ludewig, H., Sharma, S. V., Connolly, J. A., Dhaliwal, J., Wang, Y., Unsworth, W. P., Taylor, R. J. K., McLachlan, M. M. W., Shanahan, S., Naismith, J. H. \& Goss, R. J. M. (2019). Nat. Chem. 11, 1091-1097.

Hammer, P. E., Hill, D. S., Lam, S. T., Van Pée, K.-H. \& Ligon, J. M. (1997). Appl. Environ. Microbiol. 63, 2147-2154.

Heemstra, J. R. \& Walsh, C. T. (2008). J. Am. Chem. Soc. 130,1402414025.

Holm, L. (2019). Bioinformatics, 35, 5326-5327.

Ismail, M., Frese, M., Patschkowski, T., Ortseifen, V., Niehaus, K. \& Sewald, N. (2019). Adv. Synth. Catal. 361, 2475-2486.

Kabsch, W. (2010). Acta Cryst. D66, 125-132.

Keegan, R. M. \& Winn, M. D. (2007). Acta Cryst. D63, 447-457.

Keller, S., Wage, T., Hohaus, K., Hölzer, M., Eichhorn, E. \& van Pée, K.-H. (2000). Angew. Chem. Int. Ed. 39, 2300-2302.

Krissinel, E. \& Henrick, K. (2007). J. Mol. Biol. 372, 774-797.

Liebschner, D., Afonine, P. V., Baker, M. L., Bunkóczi, G., Chen, V. B., Croll, T. I., Hintze, B., Hung, L.-W., Jain, S., McCoy, A. J., Moriarty, N. W., Oeffner, R. D., Poon, B. K., Prisant, M. G., Read, R. J., Richardson, J. S., Richardson, D. C., Sammito, M. D., Sobolev, O. V., Stockwell, D. H., Terwilliger, T. C., Urzhumtsev, A. G., Videau, L. L., Williams, C. J. \& Adams, P. D. (2019). Acta Cryst. D75, 861-877.

Lingkon, K. \& Bellizzi, J. J. (2020). ChemBioChem, 21, 1121-1128.

Livingstone, C. D. \& Barton, G. J. (1993). Comput. Appl. Biosci. 9, $745-756$.

Luhavaya, H., Sigrist, R., Chekan, J. R., McKinnie, S. M. K. \& Moore, B. S. (2019). Angew. Chem. Int. Ed. 58, 8394-8399.

Ma, L., Zhang, W., Zhu, Y., Zhang, G., Zhang, H., Zhang, Q., Zhang, L., Yuan, C. \& Zhang, C. (2017). Appl. Biochem. Biotechnol. 101, 6123-6136.

Madeira, F., Park, Y. M., Lee, J., Buso, N., Gur, T., Madhusoodanan, N., Basutkar, P., Tivey, A. R. N., Potter, S. C., Finn, R. D. \& Lopez, R. (2019). Nucleic Acids Res. 47, W636-W641.

McCoy, A. J., Grosse-Kunstleve, R. W., Adams, P. D., Winn, M. D., Storoni, L. C. \& Read, R. J. (2007). J. Appl. Cryst. 40, 658-674.

Menon, B. R. K., Brandenburger, E., Sharif, H. H., Klemstein, U., Shepherd, S. A., Greaney, M. F. \& Micklefield, J. (2017). Angew. Chem. Int. Ed. 56, 11841-11845.

Menon, B. R. K., Latham, J., Dunstan, M. S., Brandenburger, E., Klemstein, U., Leys, D., Karthikeyan, C., Greaney, M. F., Shepherd, S. A. \& Micklefield, J. (2016). Org. Biomol. Chem. 14, 9354-9361.

Minges, H., Schnepel, C., Böttcher, D., Weiss, M. S., Spross, J., Bornscheuer, U. T. \& Sewald, N. (2020). ChemCatChem, 12, 818831.
Mori, S., Pang, A. H., Thamban Chandrika, N., Garneau-Tsodikova, S. \& Tsodikov, O. V. (2019). Nat. Commun. 10, 1255.

Moritzer, A.-C., Minges, H., Prior, T., Frese, M., Sewald, N. \& Niemann, H. H. (2019). J. Biol. Chem. 294, 2529-2542.

Moritzer, A.-C. \& Niemann, H. H. (2019). Protein Sci. 28, 2112 2118.

Murshudov, G. N., Skubák, P., Lebedev, A. A., Pannu, N. S., Steiner, R. A., Nicholls, R. A., Winn, M. D., Long, F. \& Vagin, A. A. (2011). Acta Cryst. D67, 355-367.

Neubauer, P. R., Widmann, C., Wibberg, D., Schröder, L., Frese, M., Kottke, T., Kalinowski, J., Niemann, H. H. \& Sewald, N. (2018). PLoS One, 13, e0196797.

Neumann, C. S., Walsh, C. T. \& Kay, R. R. (2010). Proc. Natl Acad. Sci. USA, 107, 5798-5803.

Ortega, M. A., Cogan, D. P., Mukherjee, S., Garg, N., Li, B., Thibodeaux, G. N., Maffioli, S. I., Donadio, S., Sosio, M., Escano, J., Smith, L., Nair, S. K. \& van der Donk, W. A. (2017). ACS Chem. Biol. 12, 548-557.

Pang, A. H., Garneau-Tsodikova, S. \& Tsodikov, O. V. (2015). J. Struct. Biol. 192, 349-357.

Potterton, L., Agirre, J., Ballard, C., Cowtan, K., Dodson, E., Evans, P. R., Jenkins, H. T., Keegan, R., Krissinel, E., Stevenson, K., Lebedev, A., McNicholas, S. J., Nicholls, R. A., Noble, M., Pannu, N. S., Roth, C., Sheldrick, G., Skubak, P., Turkenburg, J., Uski, V., von Delft, F., Waterman, D., Wilson, K., Winn, M. \& Wojdyr, M. (2018). Acta Cryst. D74, 68-84.

Robert, X. \& Gouet, P. (2014). Nucleic Acids Res. 42, W320-W324.

Runguphan, W. \& O'Connor, S. E. (2013). Org. Lett. 15, 2850 2853.

Seibold, C., Schnerr, H., Rumpf, J., Kunzendorf, A., Hatscher, C., Wage, T., Ernyei, A. J., Dong, C., Naismith, J. H. \& van Pée, K.-H. (2006). Biocatal. Biotransformation, 24, 401-408.

Shepherd, S. A., Menon, B. R. K., Fisk, H., Struck, A. W., Levy, C., Leys, D. \& Micklefield, J. (2016). ChemBioChem, 17, 821-824.

Thapa, H. R., Lail, A. J., Garg, N. \& Agarwal, V. (2018). Methods Enzymol. 604, 333-366.

Waterhouse, A. M., Procter, J. B., Martin, D. M. A., Clamp, M. \& Barton, G. J. (2009). Bioinformatics, 25, 1189-1191.

Yamanaka, K., Ryan, K. S., Gulder, T. A. M., Hughes, C. C. \& Moore, B. S. (2012). J. Am. Chem. Soc. 134, 12434-12437.

Yeh, E., Blasiak, L. C., Koglin, A., Drennan, C. L. \& Walsh, C. T. (2007). Biochemistry, 46, 1284-1292.

Yeh, E., Cole, L. J., Barr, E. W., Bollinger, J. M., Ballou, D. P. \& Walsh, C. T. (2006). Biochemistry, 45, 7904-7912.

Yeh, E., Garneau, S. \& Walsh, C. T. (2005). Proc. Natl Acad. Sci. USA, 102, 3960-3965.

Zeng, J., Lytle, A. K., Gage, D., Johnson, S. J. \& Zhan, J. (2013). Bioorg. Med. Chem. Lett. 23, 1001-1003.

Zeng, J. \& Zhan, J. (2011). Biotechnol. Lett. 33, 1607-1613.

Zhu, X., De Laurentis, W., Leang, K., Herrmann, J., Ihlefeld, K., van Pée, K.-H. \& Naismith, J. H. (2009). J. Mol. Biol. 391, 74-85. 